\title{
A Muscle Fibre Conduction Velocity Tracking ASIC for Local Fatigue Monitoring
}

\author{
Ermis Koutsos, Student Member, IEEE, Cretu Vlad and Pantelis Georgiou, Senior Member, IEEE
}

\begin{abstract}
Electromyography analysis can provide information about a muscle's fatigue state by estimating Muscle Fibre Conduction Velocity (MFCV), a measure of the travelling speed of Motor Unit Action Potentials (MUAPs) in muscle tissue. MFCV better represents the physical manifestations of muscle fatigue, compared to the progressive compression of the myoelectic Power Spectral Density, hence it is more suitable for a muscle fatigue tracking system. This paper presents a novel algorithm for the estimation of MFCV using single threshold bit-stream conversion and a dedicated ASIC for its implementation, suitable for a compact, wearable and easy to use muscle fatigue monitor. The presented ASIC is implemented in a commercially available AMS $0.35 \mu \mathrm{m}$ CMOS technology and utilizes a bit-stream crosscorrelator that estimates the conduction velocity of the myoelectric signal in real time. A test group of 20 subjects was used to evaluate the performance of the developed ASIC, achieving good accuracy with an error of only $3.2 \%$ compared to Matlab.
\end{abstract}

Index Terms-MFCV, ASIC, bit-stream, conduction velocity, muscle fatigue, fatigue tracking.

\section{INTRODUCTION}

An electromyogram (EMG) is a graphical representation of electrical currents associated with muscular action. Muscle contractions give rise to electrical currents which in turn form the EMG signal. The term Electromyography was introduced in 1890 by Etienne-Jules Marey who was the first to record EMG signals [1]. The EMG signal appears random in nature and has an amplitude distribution in the range of $0-10 \mathrm{mV}$ prior to amplification and a frequency bandwidth of 10-500 $H z$. An EMG segment of a static contraction is shown in Fig. 1(b).

EMG may be detected either by a needle or surface electrodes. The signal detected is called a myoelectric (ME) signal, and when recorded with a surface electrode, is called surface EMG (sEMG). sEMG monitoring is preferred to a needle electrode because of its non-invasive property in relation to human subjects. Analysis of EMG signals has been shown to be vital for several biomedical and clinical applications. Such may include medical research, rehabilitation, ergonomics, biomechanics and sports science applications. Rehabilitation is identified as one of the most important application areas of EMG signal analysis [1]. Of particular importance is its capacity to provide information about muscle fatigue.

Power spectral analysis of the ME signal is primarily used for muscle fatigue analysis. Localized muscle fatigue

E. Koutsos, V. Cretu and P. Georgiou are with the Department of Electrical and Electronic Engineering, Centre of Bio-Inspired Technology, Institute of Biomedical Engineering, Imperial College London, SW7 2AZ, U.K. (e-mail: ekoutsos@imperial.ac.uk; vlad.cretu13@imperial.ac.uk; pantelis@imperial.ac.uk).
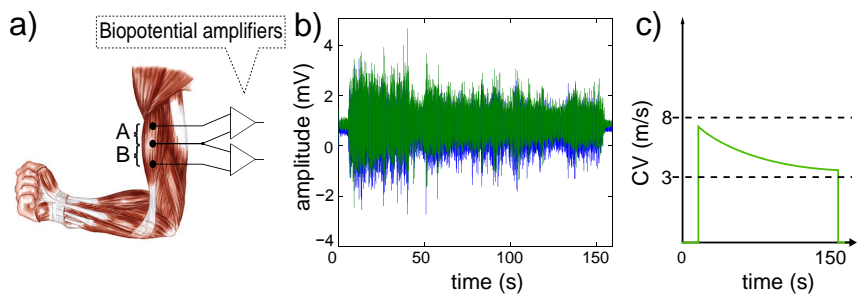

Fig. 1. Surface EMG signal path for MFCV estimation. a) Front end amplification, b) detected signals from bipolar electrodes, c) Estimation of MFCV by computing the delay between the two detected signals.

causes the spectrum to shift and compress from higher to lower frequencies [2]. This spectral change can be partially attributed to the change in conduction velocity of the Motor Unit Action Potentials (MUAPs) along the muscle fibre [3][10]. As the muscle fatigues, lactic acid and $K^{+}$accumulates in the extracellular muscle space, impairing conduction of the action potentials across the muscle membrane, thus slowing down MUAPs [11].

Muscle Fibre Conduction Velocity (MFCV) is a measure of the travelling speed of MUAPs in muscle tissue and is one of the most important items which reflects muscular activity [12]. As the muscle fatigues, MFCV decreases [2]. During a fatiguing contraction, MFCV will start from higher values and progressively reduce to lower values. The observed trend in MFCV decrease correlates well with the fatigue state of the observed muscle. Thus, the amplified and processed sEMG signal will result in a decreasing MFCV estimate, as shown in Fig. 1 (a), (b) and (c). One of the advantages of $\mathrm{MFCV}$ is that it is reliable under static and dynamic contractions [13]. Furthermore, MFCV is partially related to the observed spectral compression of the SEMG signal [14]. However, changes in the spectral content of the sEMG signal are disproportionately larger than decreases in MFCV. Moreover, recoveries in frequency are more rapid than lactate removal in the muscle, hence MFCV can provide a more detailed insight into muscle fatigue and muscle recovery than Power Spectral Density (PSD) monitoring alone, [13], [15]. Thus, MFCV would result in better muscle fatigue tracking than Median/Mean frequency analysis.

MFCV can be calculated using two different approaches; extracting information from one detected sEMG signal alone or comparing two or more detected sEMG signals along the muscle fibre direction(Fig. 1(a)). Estimating MFCV by processing one detected signal works by extracting spectral information from the signal. Algorithms using this approach rely on finding characteristic spectral frequencies of the $\mathrm{ME}$ signal. Lindstrom et al. [8] developed a mathematical model 


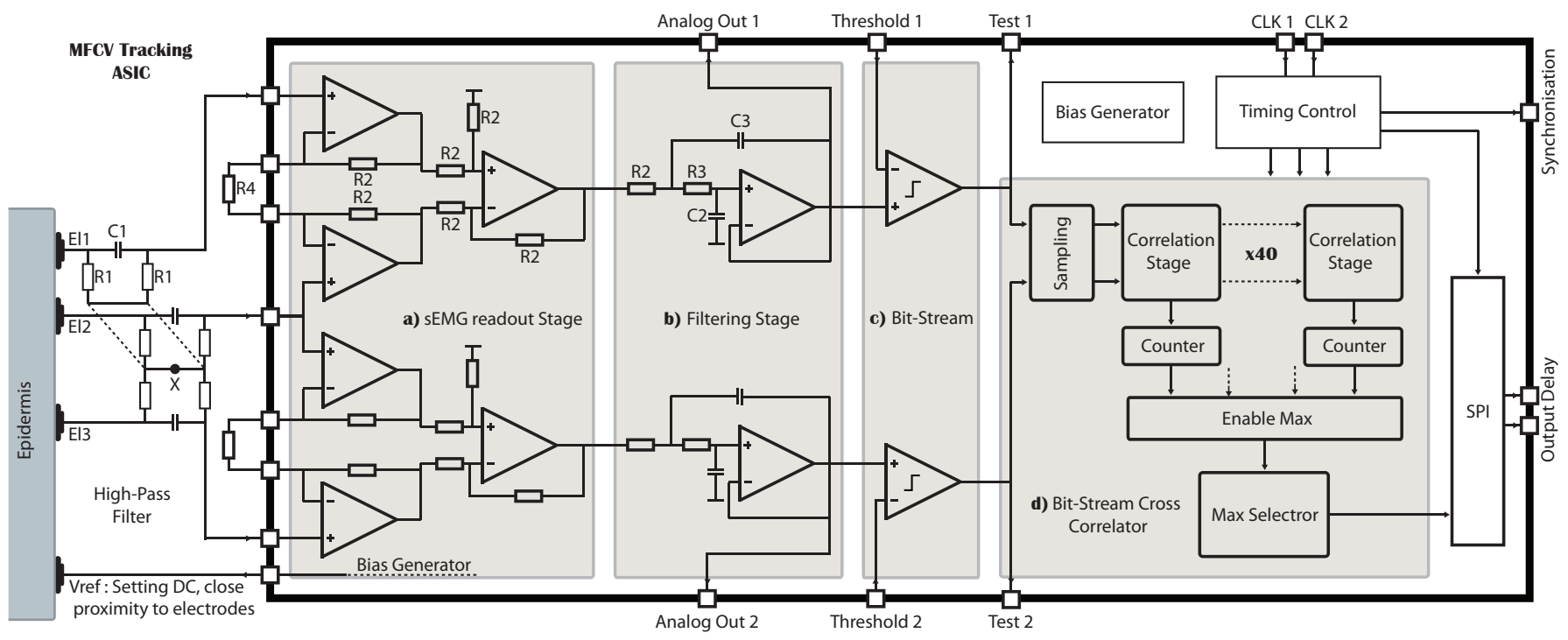

Fig. 2. System block architecture of the Muscle Fibre Conduction Velocity tracking ASIC.

linking MFCV with the observed EMG power spectrum. Thus, a shift in the power spectrum can account for a change in MFCV. The disadvantage of these methods is that they require spectral analysis tools, they are more sensitive to noise, they will introduce large variance and they are sufficiently difficult to detect accurately [14], [16]. Another approach for finding MFCV involves processing two detected sEMG signals. The electrodes are placed perpendicular to the underlying muscle fibres. Algorithms following this approach include finding the distance between reference points. This method assumes that the two detected signals are identical with the addition of noise. Thus, the two signals would have the same shape with the introduction of a delay. As a result, any specific reference point such as a valley, a peak or a zero can be used to align the two signals and estimate the delay between them. Similarly, processing signals in frequency domain eliminates the issue of time resolution. Hence the phase difference between the detected signals can be calculated to estimate MFCV. Finally, the time lag at which the crosscorrelation function is maximum can be used as an estimator of delay, as was early demonstrated for surface EMG signals by Parker et al. [17]. The underlying principle of the presented methods is the measurement of time/phase delay between two myoelectric signals [14]. CMOS based System-on-Chip (SoC) solutions show significant promise to create bespoke solutions for wearable medical devices with small form factor, low power consumption and increased accuracy. Implementing the cross-correlation method using low power digital CMOS logic shows great promise in terms terms of computational complexity, efficiency and noise immunity [6], [17]-[23].

This paper presents a novel SoC for monitoring MFCV from surface EMG signals using a real time CMOS bit-stream crosscorrelator. This is the first demonstration of a system capable of real time MFCV estimation. A low power ASIC implementation of the MFCV tracker facilitates its potential use as a wearable device dedicated for muscle fatigue monitoring. This paper is organised as follows: Section II presents the
ASIC architecture and front-end implementation, Section III demonstrates the MFCV tracking algorithm, Section IV the MFCV tracking circuit implementation, Section V the measured experimental results, and finally Section VI concludes our study.

\section{MFCV TRACKING ASIC ARCHITECTURE}

The presented MFCV ASIC shown in Fig. 2 consists of four major building blocks: a) a sEMG dual channel Instrumentation Amplifier (IA), b) two Sallen Key low-pass filters, c) a bit-stream converter comprised by two analog comparators and d) a digital bit-stream cross-correlator. In addition, the MFCV ASIC includes a bias circuit generator, a digital timing control circuit and a Serial Peripheral Interface (SPI) to communicate with any receiving circuit.

The operation of the MFCV ASIC can be described as follows: the dual IA amplifies the detected sEMG signals which are then low-pass filtered to extract the signal attributes in the required frequency band $(10 \mathrm{~Hz}-500 \mathrm{~Hz})$. Following that, the bit-stream converter digitises the sEMG signals and feeds them to the bit-stream cross-correlator that computes the time delay between them.

\section{A. sEMG Signal Processing}

The circuit has been implemented in a commercially available $0.35 \mu \mathrm{m}$ CMOS technology provided by AMS (C35B4). Standard cell libraries were used for the implementation of the operational amplifiers and comparators in stages $\mathbf{a}, \mathbf{b}$ and $\mathbf{c}$ as the focus of this research is the bit-stream cross-correlation algorithm application for MFCV tracking and system implementation.

Instrumentation Amplifiers (IA) should be capable of rejecting up to $300 \mathrm{mV}$ DC Polarization Voltage (PV) from the biopotential electrodes [24], [25]. Thus, ac coupling is needed to avoid saturating the output due to input DC offsets. Existing architectures that can implement rejection of such 
high DC voltages without using off-the-shelf components lead to lower performance IAs [26], [27] or suffer from trade-off's resulting in reduced PV [28]-[30]. On the other hand, the use of conventional, simple, off-chip high-pass filters significantly reduces the input impedance, where especially the common mode input impedance is very important for achieving high Common Mode Rejection Ratio (CMRR).

Thus, an external floating high-pass filter is used, as shown in Fig. 2. The intended application of the ASIC as a wearable device allows the use of external components. The main advantage of floating high-pass filter compared to conventional passive high-pass filters is the elimination of the grounded resistor, implementing very large common mode input impedance [24], [25]. With $R 1=10 M \Omega$ and $C 1=1.5 n F$ the resulting cutoff is $10.6 \mathrm{~Hz}$ to best filter out motion artefacts [31]. However, this filter structure requires a fourth electrode to bias the input filter structure and set the DC voltage of the body around the electrodes. This reference DC level must satisfy the amplifier's input common mode range requirements and is set at a voltage $V_{\text {ref }}=V_{\text {supply }} / 2$. The common mode voltage of the detected sEMG signal feeding the amplifiers is the averaged DC electrodes (1-3) voltage at node $\mathbf{X}$ (Fig. 2). Since the input network is not grounded, when a common mode input voltage is applied, no currents flow through the network.

The low-pass filter of Sallen Key topology is implemented with a cutoff of $2.5 \mathrm{kHz}$, with $R 2=100 \mathrm{~K} \Omega, R 3=1 \mathrm{M} \Omega$ and $C 2, C 3=33 p F$. The structure is duplicated to allow two channel operation. The reference voltages of the two comparators are kept separate to allow offset mismatch compensation. Furthermore, the feedback resistors responsible for the amplifier gain $(R 4)$ were not implemented on the IC, but left to be completed with external components to allow gain flexibility.

\section{MFCV TRACKING AlgORITHM}

Cross correlation requires two signals to estimate the delay between them. The sEMG detection points must lie in the muscle fibre path, from the innervation zone to the myotendinous part of the muscle. The innervation zone is a small region or band of muscle tissue wherein MUAPs originate and then propagate bidirectionally toward each tendon [32]. The two required sEMG signals are detected differentially between points $\mathbf{A}, \mathbf{B}$ and are shown in Fig. 1(a). Despite all of its advantages in MFCV estimation, cross-correlation is a computationally intensive time-domain operation because of the large amount of multiplications required. Cross-correlation between two signals $(x, y)$ is described by the following equation, Eq. 1:

$$
r_{l}=\sum_{i=1}^{n} x(i) y(i \quad l),
$$

where $l$ is the time shift between the two signals being correlated. For every point in time, all the samples (n) in the correlation window must be multiplied and accumulated. Implementing a cross-correlator following this approach requires

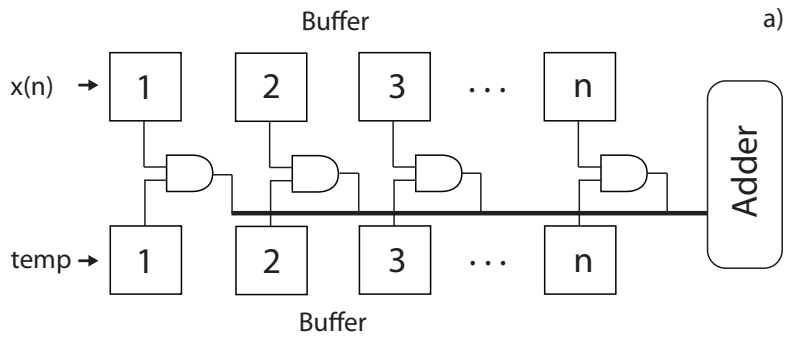

a)

b)

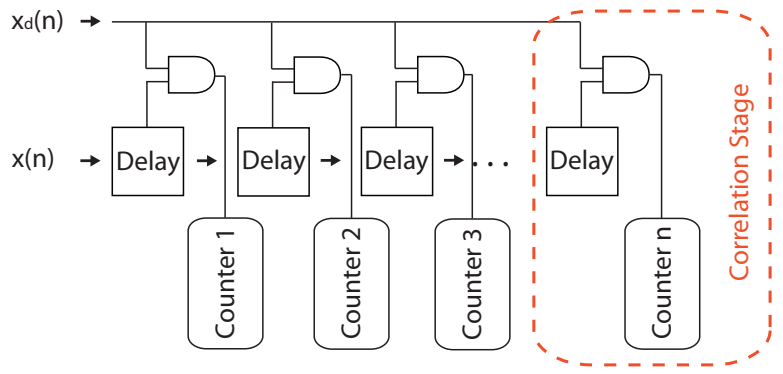

Fig. 3. Comparing architectures: a) Lande et al. bit-stream correlator, b) proposed bit-stream correlator.

two signal buffers, multipliers and would result in a power hungry system.

Cross-correlation is estimating the time delay between the two signals by utilizing the complete sEMG waveforms. The sEMG signal is a superposition of many MUAPs from different muscle fibres, resulting to a spike based signal. As a result, any specific reference point such as a valley, a peak or a zero can be used to align the two signals and estimate the delay between them. Thus, cross-correlation can be applied to finding the distance between reference points, thus simplifying the cross correlation process. The sEMG signals can be converted to a discrete signal with the aid of a single threshold. Our work demonstrates that single threshold is an adequate alternative to digitizing the complete sEMG signal, while retaining the necessary information for cross correlation and delay estimation. The novelty of this research work lies in eliminating the need of cross-correlating the whole sEMG signal, by cross-correlating a single bit approximation of it. Thus, complicated cross-correlator architectures can be replaced by simple, bit-stream cross-correlator designs. Lande et al. [33] replaced the complicated multiplications required for cross-correlation by AND gates. The input signal is a single bit stream coming from a sigma delta modulator. In this approach the input signal is buffered and similar to Eq. 1, for every time shift the buffer window is correlated with a template signal. However, this approach requires a very large sampling window. Matlab simulations revealed that buffer lengths over 10kbits are needed for accurate results. Consider Fig. 3(a) for a graphical representation of the algorithm described above.

The two EMG signals are converted to a bit-stream with the use of a comparator. The aim of the MFCV cross-correlator is to estimate the time delay between the two bit-stream signals $x(n)$ and $x_{d}(n) . x(n)$ is the digitized EMG signal detected at point A (see Fig. 1(a)) and $x_{d}(n)$ is a delayed version of $x(n)$ detected at point $\mathbf{B}$. Thus, there is no need to buffer $x_{d}(n)$ for cross-correlation. In the proposed architecture of the bit- 
stream correlator, the bit-stream buffer window is eliminated by continuously correlating the two signals in a given time window. This is achieved by counting all the time instances where the two signals are the same. Hence, a correlation time window replaces the buffer window for $x(n)$. The proposed bit-stream architecture is similar to the operation of correlator banks [34]. However, this would return the similarity of the two signals for a given time lag. Discrete time lags for the cross-correlation output are obtained by continuously delaying the input signal and repeating the aforementioned process. Thus, obtaining a correlation result for every discrete time delay. Finally, the time lag between the two signals is returned by the counter with the larger value. This approach greatly simplifies the number of transistors required. However, the time lag resolution is limited. The time lag dynamic range depends on the number of time delays introduced to the system. Furthermore, the resolution of the system can be varied according the sampling frequency $(T=$ Delay $)$ and the cross-correlation time window. Consider Fig. 3(b) for the proposed bit-stream correlator which comprises of several correlation stages, where each stage has a delay block, a counter and a correlator.

\section{BIT-STREAM CROSS-CORRELATOR}

\section{A. Correlation Stage}

As mentioned in the Section III, the bit-stream correlator is made of several correlation stages connected in series. Each stage comprises of a delay block, a counter and a correlator. The D-type Flip Flop (DFF) acts as a delay block, and the delay time is controlled by the sampling frequency of the system $(C L K 1)$. A 14 bit ripple counter was found to be enough to meet the requirements of the system. The counter size was selected by analysing retrospective sEMG data to allow operation with correlation time windows over 1 second and high sampling frequencies $(C L K 1>=10 \mathrm{kHz})$. The use of XNOR gates as a bit correlator improves the original AND gate design by taking all possible digital cases into consideration. The circuit schematic is shown in Fig. 6 . A second clock $(R S T)$ with a much lower frequency than $C L K 1$ is used to reset the counters and thus define the crosscorrelation time window.

In the case presented, $x_{d}(n)$ is signal1 and $x(n)$ is signal2. signal2_del will feed signal2 of subsequent stages. A total of 40 correlation/delay stages were introduced to the system, providing a wide range of time delay estimation. The dynamic range of the system can be varied according the sampling frequency $C L K 1$ and the delay estimation accuracy according to the cross-correlation time window $(R S T)$. In order to characterise the system, it was modelled in Simulink and compared with Matlab xcorr function using real sEMG data from the biceps brachii muscle of a test subject. Fig. 4 shows the Mean Absolute Relative Difference between the modelled system and Matlab simulations for a varying comparator threshold. It can be seen that as the threshold is swept above the common mode, the relative error increases. Hence, a realistic ASIC implementation would require a threshold level as low as possible whilst above the noise floor. Fig. 5 shows a parametric simulation with the sampling frequency (CLK1) and correlation

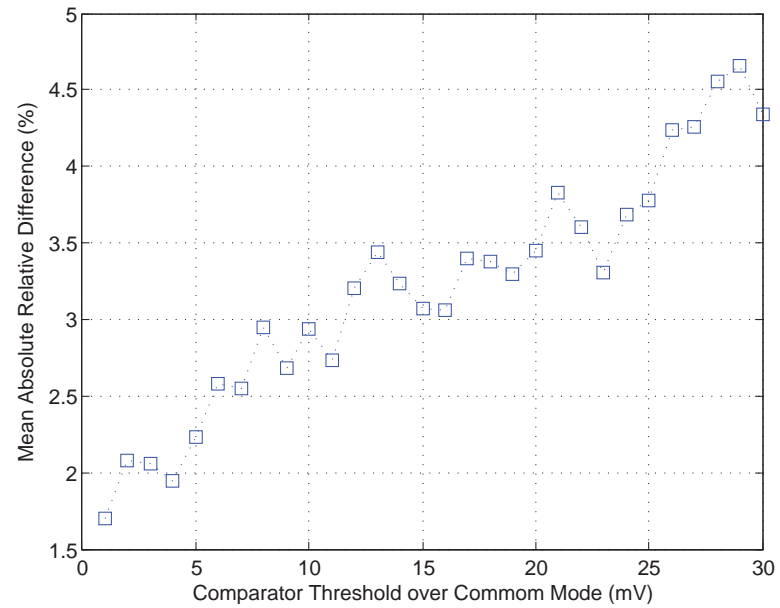

Fig. 4. Mean Absolute Relative Difference between Matlab xcorr function and modelled system with a varying threshold.

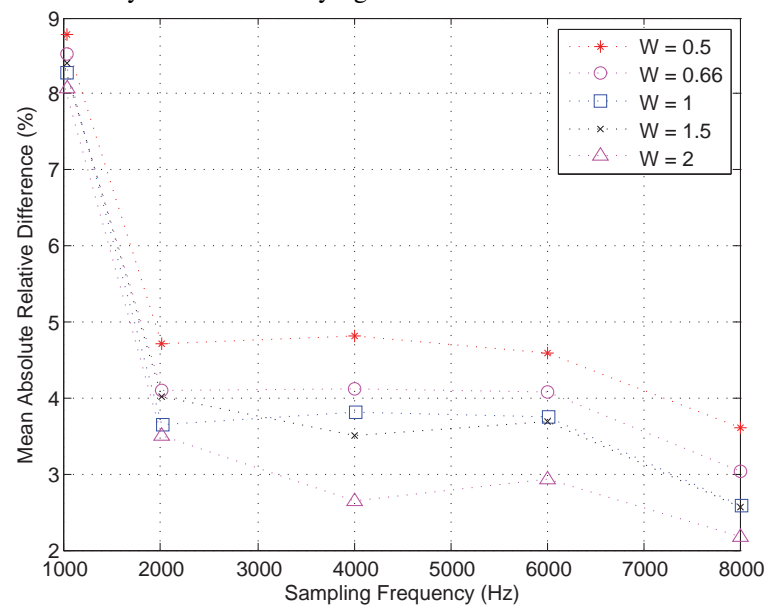

Fig. 5. Mean Absolute Relative Difference between Matlab xcorr function and modelled system, where $W$ is the correlation window.

window as variables. Increasing the sampling frequency results to higher accuracy but limits the dynamic range of the system. The results show that sEMG bit-stream cross-correlation is an accurate representation of cross-correlation which allows it to used for tracking MFCV. A longer correlation window would yield better delay estimation accuracy but would result to a slowly tracking system. The proposed approach of this bitstream cross-correlator resembles the basic architecture of a time-to-digital converter. According to [35], the measurement interval $\Delta T$ in a time-to-digital converter can be expressed as in Eq. 2, where $T_{c}$ is the period of $C L K 1$. The error in the measured time interval $\Delta T$ is $\epsilon_{r}$ and can be equal to twice the clock period. Thus, a delay chain length of 40 yields a relative error of $5 \%$, which is considered acceptable for the intended application.

$$
\begin{aligned}
& \Delta T=N T_{c}+\epsilon_{r} \\
& \epsilon_{r} \quad\left[T_{c} ;+T_{c}\right]
\end{aligned}
$$

Fig. 7 shows the signal propagation in the system. Fig. 7(a) shows an EMG signal segment as detected by the biopotential front end amplifiers. Fig. 7(b) shows the resulting bit-stream after single bit digitization. Fig. 7(c) and (d) show the propagation of the bit-stream trough the delay blocks of the system. 


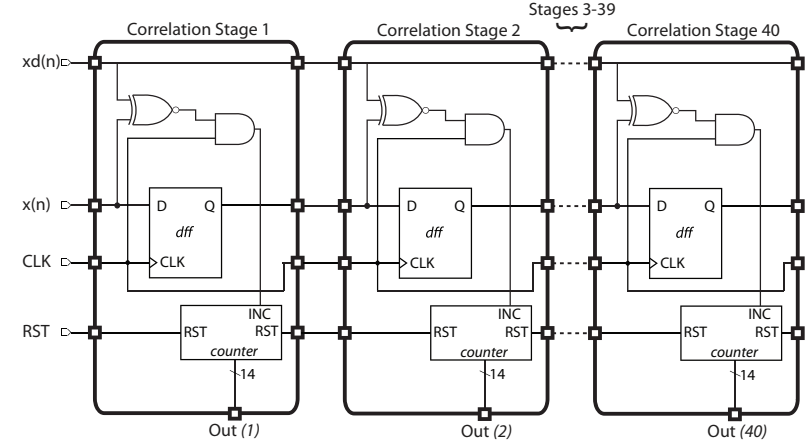

Fig. 6. Correlation stage with delay block, counter and correlator (XNOR).

Finally, Fig. 7(e) and (f) show the correlation results and how these are interpreted by the counters in real time. At the end of the correlation time window, the maximum value counter is selected. The delay this counter is linked to, represents the time lag between the input signals. After that, the counters are reset to zero and correlation starts again.

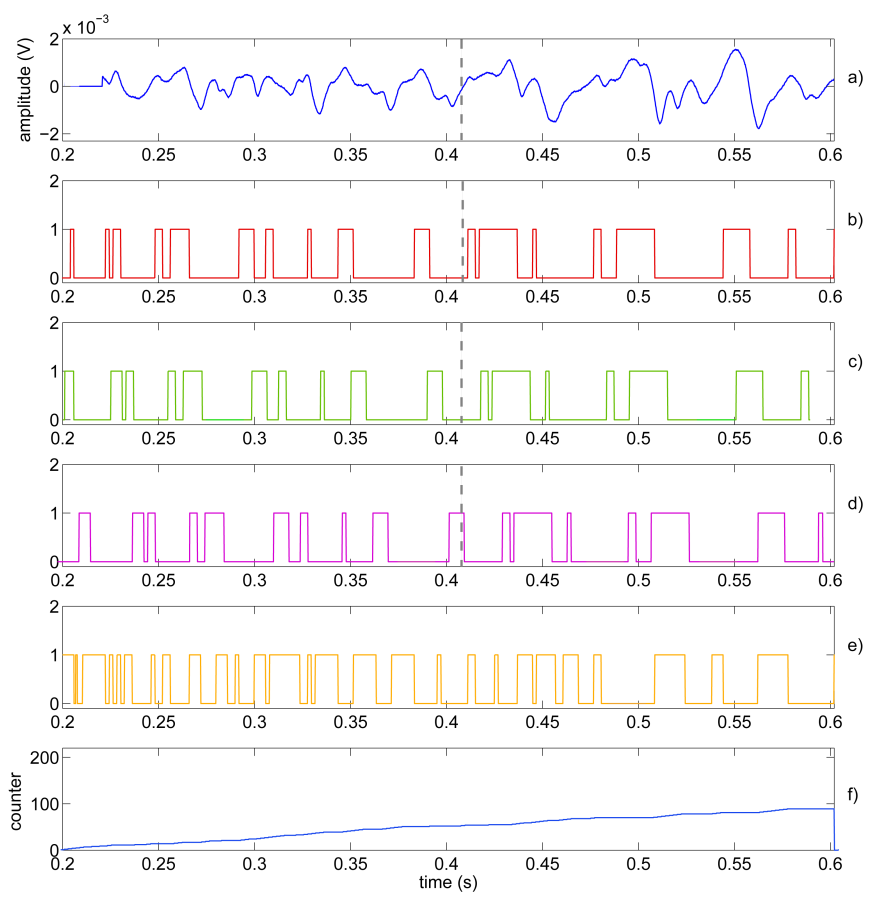

Fig. 7. Correlation stage signals from simulations: a) EMG signal, b) $x_{d}(n)$ bit-stream, c) $x(n)$ bit-stream delayed by 10 previous correlation stages, d) $x(n)$ bit-stream delayed by all previous stages, e) XNOR bit correlation result between $b$ ) and c), e) counter result after adding all XNOR values in real time.

\section{B. Maximum Detector Stage}

At the end of the correlation time window, all the counters of the system are read. The correlation stage (i.e. delay) of the counter with the maximum value best represents the time lag between the two input signals, as shown in Eq. 3. A maximum function compares all the counters in cycles. The maximum function consists of smaller blocks, each comparing two 14 bit numbers, and operates using sequential logic. It starts by comparing all the results in pairs and then proceeds with evaluating the results of the last comparison. The maximum function operates using sequential logic and is shown in Fig. 8. Every maximum operation returns a binary flag, which passes down to the next comparison and indicates which of the two compared numbers is the maximum. Thus, a binary one means the first of the two numbers is bigger. This way, the counter position number (hence delay number) and not the counter value is returned when the operation is finished. Consider Eq. 4 as an example for calculating the counter path.

$$
D_{n, i}=\left.\max \left(C_{1}, C_{2} \ldots C_{n}\right)\right|_{t=i}
$$

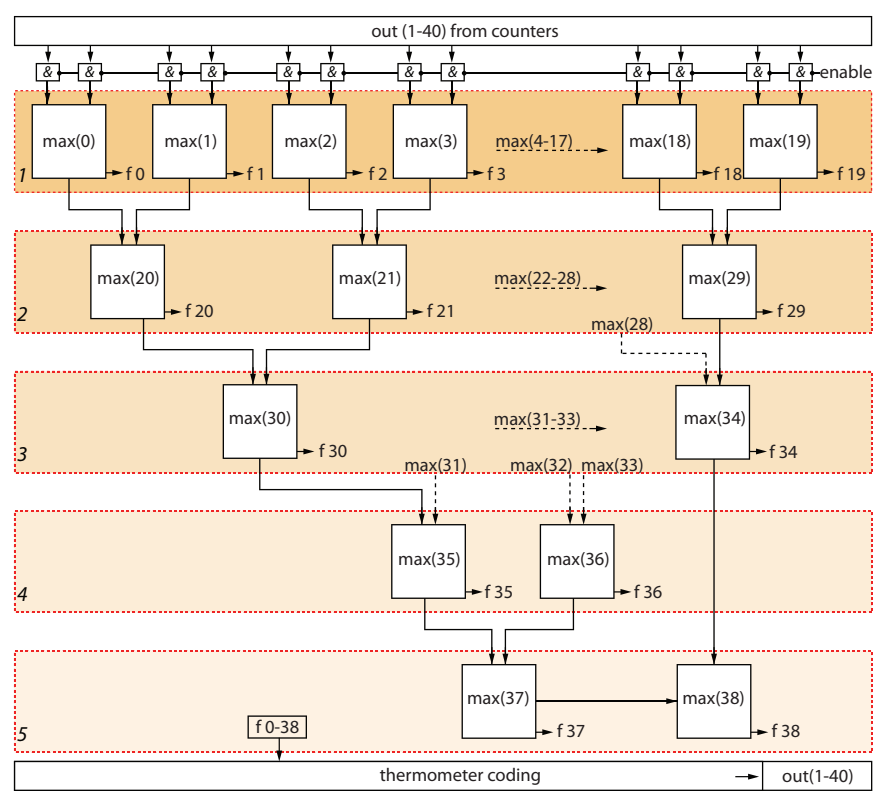

Fig. 8. Maximum Function Flow.

$$
\begin{aligned}
& \operatorname{out}(1)=f_{38} \quad f_{37} \quad f_{35} \quad f_{30} \quad f_{20} \quad f_{0} \\
& \operatorname{out}(2)=f_{38} \quad f_{37} \quad f_{35} \quad f_{30} \quad f_{20} \quad \bar{f}_{0} \\
& \operatorname{out}(3)=f_{38} \quad f_{37} \quad f_{35} \quad f_{30} \quad \bar{f}_{20} \quad f_{1}
\end{aligned}
$$

\section{EXPERIMENTAL RESUlTS}

The MFCV tracking ASIC micro-photograph is shown in Fig. 9. The layout area for the digital core is $1122 \mu \mathrm{m}$ by $494 \mu \mathrm{m}$ and for the analog front end is $800 \mu \mathrm{m}$ by $500 \mu \mathrm{m}$, with a total area of $1 \mathrm{~mm}^{2}$. The total static and dynamic power consumption of the digital core (clocked at $10 \mathrm{kHz}$ ) is $1 \mu \mathrm{W}$ and of the analog front end is $2.071 \mathrm{~mW}$ from a $3.3 \mathrm{~V}$ supply. The power consumption of the analog front end includes the power of two IAs, two high-pass filters and two bit-stream converters. Although more power efficient designs exist, they are not required for the intended purposes of this application, as the current power consumption is sufficient for a wearable sEMG device [25], [27]. Table I summarises the size and power consumption values. 


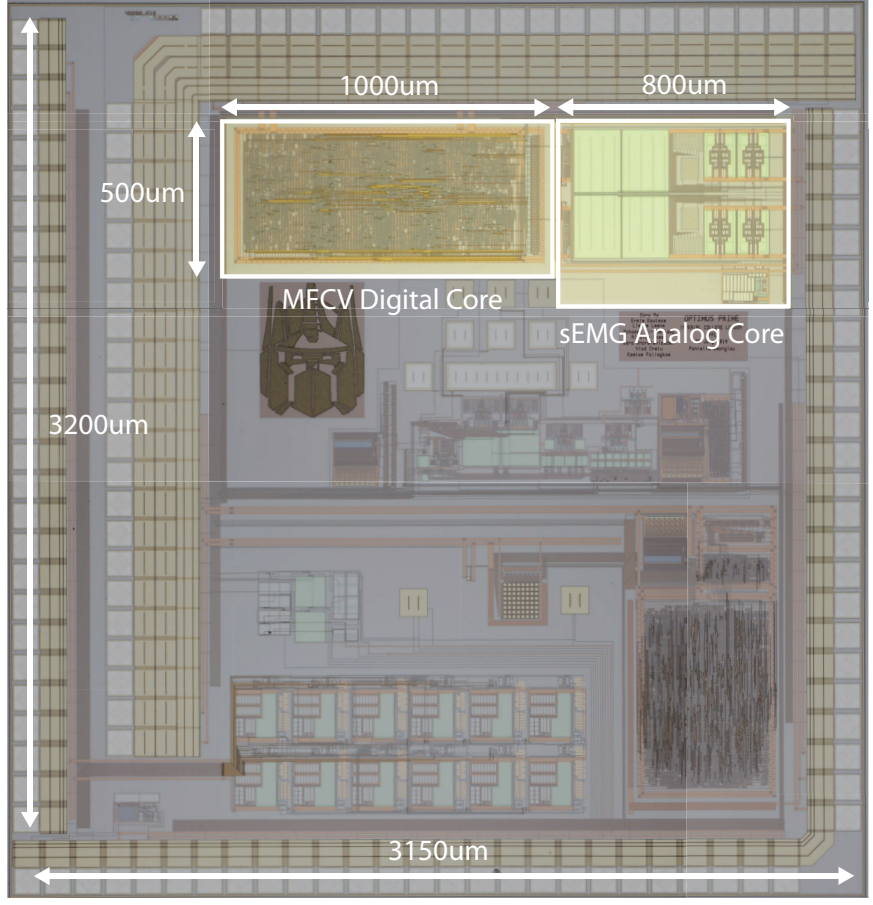

Fig. 9. Die micro-photograph of the MFCV tracking ASIC.

TABLE I

MFCV ASIC SIZE AND POWER BREAKDOWN.

\begin{tabular}{ccc}
\hline Core & Measured Current & Size \\
Analog & $616 \mu \mathrm{A}$ & $0.4 \mathrm{~mm}^{2}$ \\
Digital & $290 \mathrm{nA}$ & $0.554 \mathrm{~mm}^{2}$ \\
Biasing & $11.5 \mu \mathrm{A}$ & - \\
\hline
\end{tabular}

\section{A. System Validation}

The characterization of the IA is performed at the output of the sEMG readout stage. Fig. 10 shows that the measured integrated input-referred noise of the IA is $3.579 \mu V$ with a noise floor at $135 \frac{\mu V}{\overline{H z}}$. sEMG has a range of $50 \mu V$ to 10 $m V$. Thus, the presented front end biopotential amplifier is more that adequate to amplify sEMG signals. Fig. 11 shows the measured differential and common-mode gain of the IA followed by the high-pass filter. The external floating low pass filter was included during measurements. The resulting Common Mode Rejection Ratio (CMRR) is $85.24 d B$ in the bandwidth of interest.

Following that, in order to verify the functionality of the system, the front end amplifier was disconnected from the digital core. Thus, the bit-stream cross correlator was tested on its own. Two square signals of amplitude $3.3 V_{p-p}$ and frequency $100 \mathrm{~Hz}$ were placed on the inputs of Channel 1 and Channel 2. The aim was to resemble a prefect case scenario of amplified and digitized sEMG signals. The reset clock $(C L K 2)$ of the system was set to $1 \mathrm{~Hz}$ to maximize the correlation window accuracy. The sampling clock $(C L K 1)$ was set to $10 \mathrm{kHz}$. Starting from a phase delay between the two pulses of $0 \mathrm{sec}$, it was gradually increased all the way to $3.9 \mathrm{msec}$ with $1 \mu \mathrm{s}$ increments. The measured delay appeared on the output in the form of a step ladder. The mean time to switch from one unit delay to another was $101.3 \mu \mathrm{s}$, close to

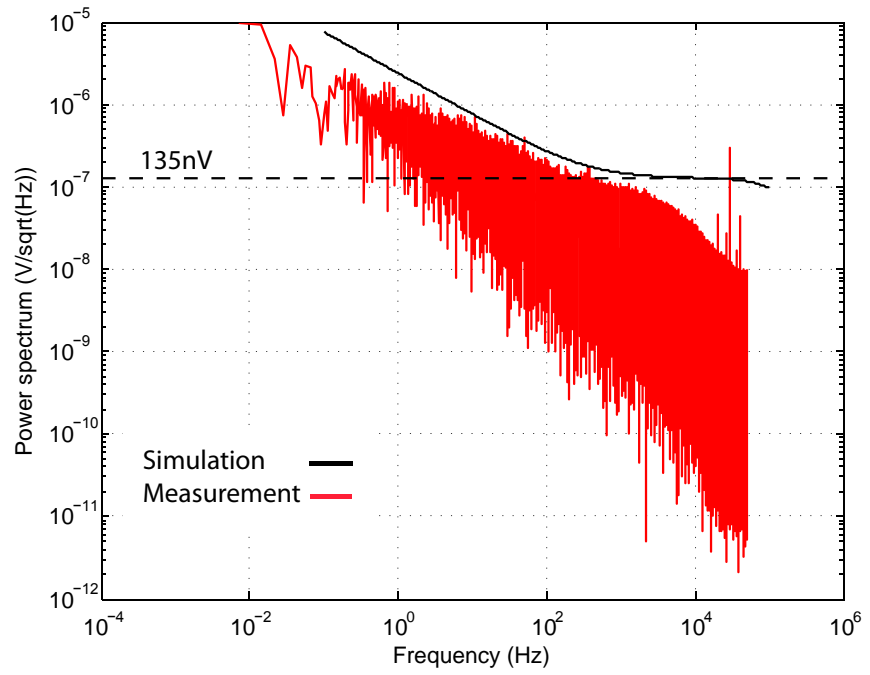

Fig. 10. Input-referred noise measurement and simulation of the IA chan. 1.

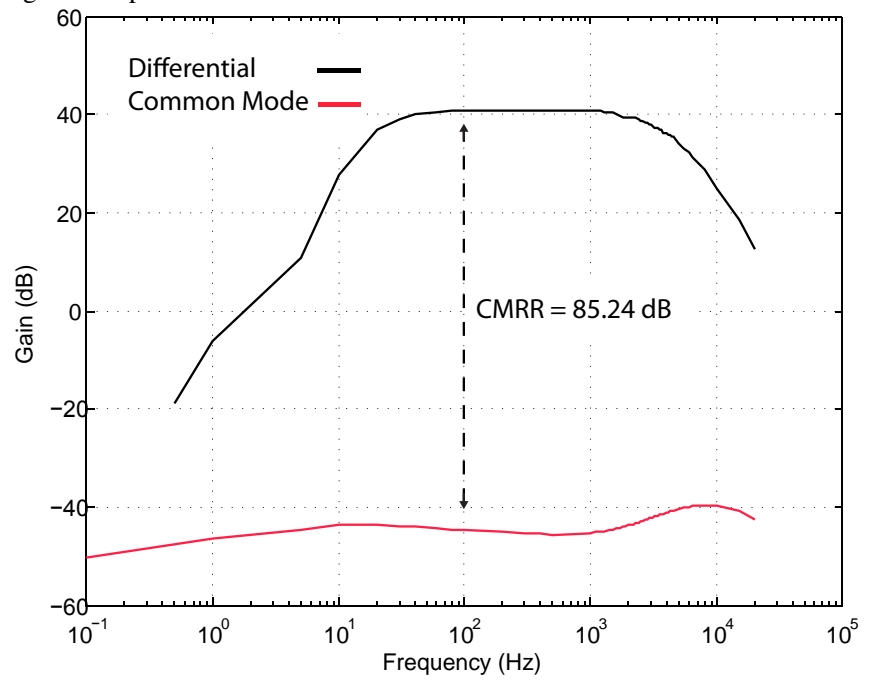

Fig. 11. Common-mode rejection ratio measurement of the IA (channel 1) using a $500 \mathrm{mVpp}$ common-mode input signal.

the ideal time of $100 \mu s$, resulting in a mean error of less that $2 \mu s$ with a standard deviation of $7.4 \mu s$.

\section{B. Validation on Human Subjects}

Twenty (20) volunteers (4 female, 16 male) were used to evaluate the performance of the MFCV tracking ASIC. This work has been approved by the Imperial College Joint Research Compliance Office (JRCO) ethics committee (ICREC ref: 15IC2481).

1) Experimental Protocol: Four disposable surface EMG electrodes were placed on the skin of the participant's arm to monitor activity of the biceps brachii muscle, according to Fig. 12. Prior to electrode placement, we prepared the skin by cleaning it with medical alcohol. Subjects were seated or standing, depending on their preference, with the back and elbow fixed against a wall in order to minimize compensatory movements. Subjects were required to keep in contact with the wall throughout the whole duration of the testing. The subject specific calibration of EMG sensors involved the performance of five Maximum Voluntary Contractions (MVCs). Following 
TABLE II

SUBJECT TRIAL RESULTS: A) SUBJECT NUMBER, B) CHANGE IN MFCV FROM CHIP, C) CHANGE IN MFCV FROM MATLAB, D) MARD AND E) MVC FORCE.

\begin{tabular}{lllllllllllllllllllll}
\hline A) $\#$ & 1 & 2 & 3 & 4 & 5 & 6 & 7 & 8 & 9 & 10 & 11 & 12 & 13 & 14 & 15 & 16 & 17 & 18 & 19 & 20 \\
B) $(\mathrm{m} / \mathrm{s})$ & 1.13 & 1.40 & 2.38 & 2.13 & 1.59 & 3.01 & 1.64 & 4.11 & 0.88 & 4.80 & 1.78 & 5.72 & 2.6 & 20.67 & 1.32 & 2.13 & 0.48 & 1.09 & 3.35 & 2.28 \\
C) $(\mathrm{m} / \mathrm{s})$ & 1.22 & 1.71 & 2.66 & 3.28 & 1.69 & 3.48 & 1.32 & 2.93 & 1.01 & 5.47 & 2.14 & 5.73 & 2.85 & 18.41 & 1.44 & 2.27 & 2.15 & 0.99 & 3.54 & 2.35 \\
D) $\%$ & 1.12 & 1.77 & 1.26 & 2.56 & 2.67 & 6.47 & 2.37 & 3.04 & 0.93 & 9.64 & 1.60 & 2.08 & 1.86 & 5.38 & 1.34 & 1.39 & 3.11 & 3.63 & 6.78 & 3.70 \\
E) $\mathrm{Kg}$ & 19.5 & 23.0 & 23.5 & 23.5 & 13.2 & 23.5 & 15.0 & 13.5 & 20.5 & 16.0 & 28.5 & 27.6 & 25.4 & 24.6 & 36.0 & 32.0 & 19.0 & 34.0 & 35.0 & 17.6 \\
\hline
\end{tabular}

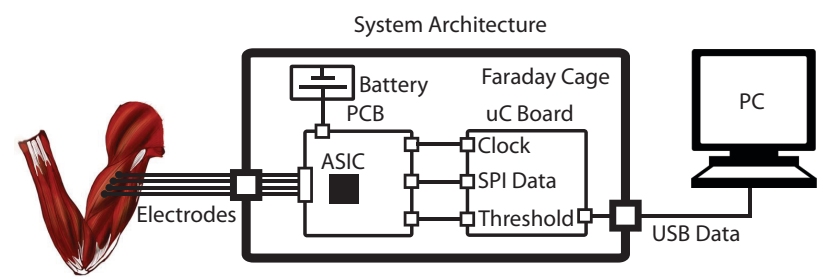

Left Bicept Brachii
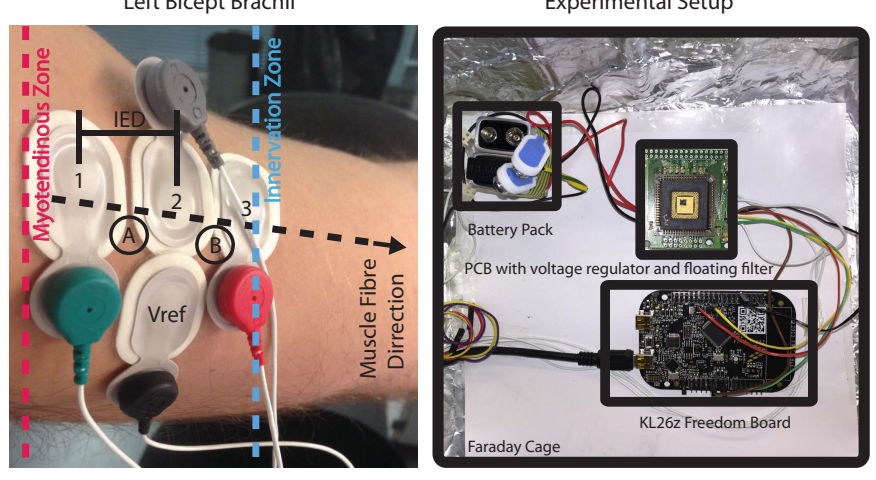

Fig. 12. Top: Architectural diagram of experimental setup. Left: Electrode configuration for bipolar single differential sEMG amplification. Muscle zones are displayed. Right: Experimental setup including micro-controller for data transmission, clock generation and threshold position. Faraday cage used in noise measurements only.

that, the subjects were asked to perform isometric contractions by pulling against a handle attached to an electronic scale. The bottom end of the scale was attached to the ground. Subjects were required to sustain a constant force, pre-set at $70 \%$ of their MVC force, for as long as they deemed possible. After the force reading dropped more than $10 \%$ of the pre-set level, the experiment would stop.

One requirement of the system is the two independent thresholds to set the levels of the bit-stream converters. The threshold level should be low enough to be closer to the base of the sEMG MUAP spike and at the same time be above the peak noise amplitude. A separate micro-controller was introduced solely as a control interface to a computer and was used to transmit the output bit-stream of the converter, set the clock frequency and adjust the thresholds accordingly. The threshold adjustment would take place prior to the start of the MFCV estimation, hence the output of the amplifier was its DC operating point. The threshold was set to a high level and gradually reduced until an empirical value of 5 crosses were detected in one second, ensuring the threshold is above $+3 \sigma(99.7 \%)$ of the noise amplitude distribution function. This algorithm would ensure that both thresholds are placed at a level just above the noise floor.

2) Results from Human Trials: To the best of our knowledge, this paper presents the first MFCV tracking ASIC for local fatigue monitoring. Thus, the accuracy of the ASIC is
TABLE III

COMPARISON BETWEEN MFCV PHYSIOLOGICAL VALUES.

\begin{tabular}{lccccccl}
\hline Literature & {$[10]$} & {$[18]$} & {$[19]$} & {$[20]$} & {$[22]$} & {$[40]$} & $\begin{array}{l}\text { This } \\
\text { work }\end{array}$ \\
& & & & & & & \\
Mean/Range & 5.1 & 4.2 & 2.6 & 4.64 & 2.53 & 4.4 & 5.4 \\
MFCV (m/s) & & & -5.3 & & -4.87 & & \\
\hline
\end{tabular}

established by a direct comparison between the ASIC and $M A T L A B^{T M}$. The amplified sEMG signal from the ASIC is recorded using a 16-bit ADC by ADInstruments. Following that, the MFCV is computed in MATLAB using the xcorr function and then compared to the ASIC estimate.

An example of the estimated MFCV from MATLAB and that measured form the ASIC from one subject is presented in Fig. 13. The subject starts the static contraction form a rested state, with an MFCV of $6 \frac{\mathrm{m}}{\mathrm{sec}}$. As the subject gets tired, the MFCV drops to lower values, until the point that the subject is completely fatigued and stops the experiment. The MFCV has reached a new lower value of $4 \frac{\mathrm{m}}{\mathrm{sec}}$.

Table II presents the change in MFCV from a rested state to a complete fatigue state of the muscle for all 20 subjects. The estimated values of MFCV decrease in all subjects during fatigue, as seen in Table II (B), (C) by the relative change in MFCV from the ASIC and $M A T L A B^{T M}$ respectively. Although MFCV is not always monotonically decreasing, the end point is at a lower value than the starting point in all subjects. The relative error between $M A T L A B^{T M}$ and the ASIC is estimated across the complete MFCV trends and not only on the relative change of MFCV and is shown in Table II (D). Time lag is converted to velocity using Eq. 5, where $C L K 1$ is the sampling frequency, OutputDelay\# is the ASIC delay estimate between $1-40$ and $I E D$ is the Inter Electrode Distance (Fig. 12, IED $=2 \mathrm{~cm}$ ). The estimated MFCV values match the physiological values reported in literature for a fatiguing isometric contraction [10], [18]-[20], [22], [40]. The reported MFCV values are compared with the MFCV values seen in this study in Table III.

The correlation window was set to 1 second $(C L K 2=$ $1 H Z$ ) to maximize the accuracy of the bit-stream crosscorrelator. The sampling/delay clock was set to $6.25 \mathrm{kHz}$ to establish a wide MFCV dynamic range. The same parameters were imported in MATLAB for analysis. The ASIC is found to have a Mean Absolute Relative Error (MARD) of 3.2\%. Subject specific MARD results are presented in Table II. The two MFCV trends (MFCV: $M A T L A B^{T M}$, MFCV: ASIC) are compared point by point and the distribution of comparison data is displayed in a box plot in Fig. 14.

$$
C V\left(\frac{m}{\text { sec }}\right)=\frac{I E D C L K 1}{\text { OutputDelay }_{\#}}
$$




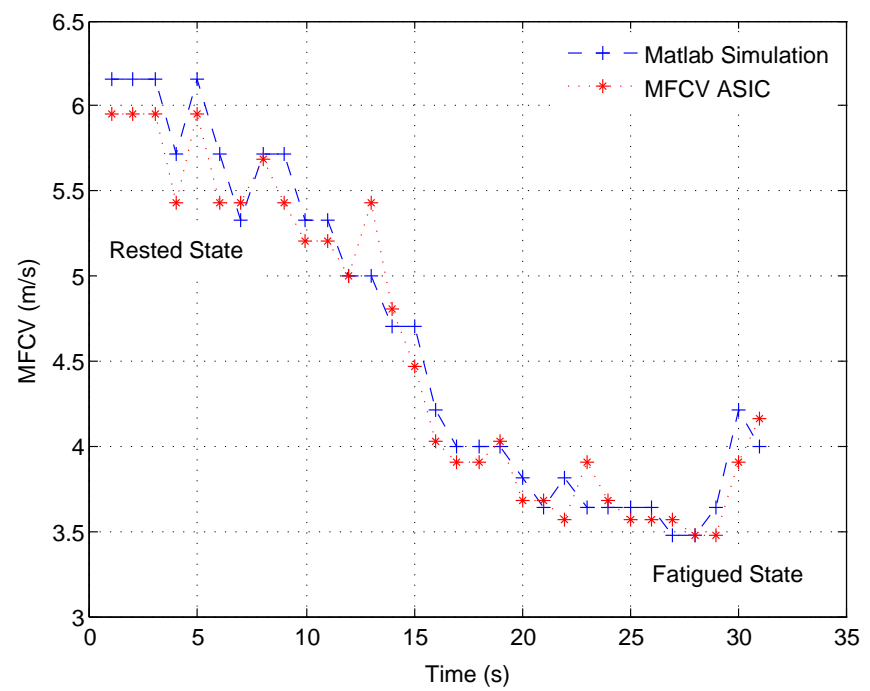

Fig. 13. MFCV trial results fro subject 11. The rested state MFCV is $6 \frac{m}{s e c}$ and progresses to $4 \frac{\mathrm{m}}{\mathrm{sec}}$ as the muscle fatigues.

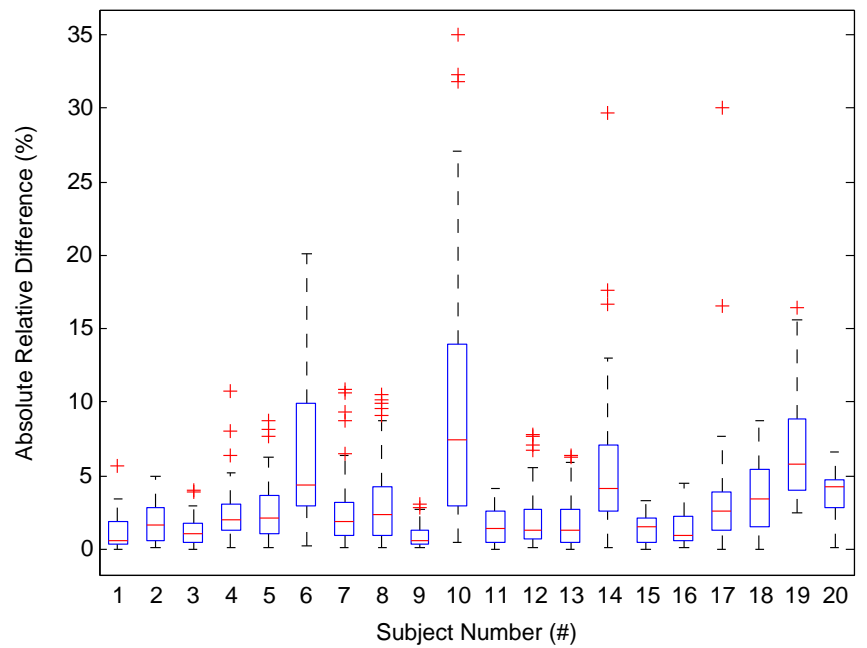

Fig. 14. Fatigue trial results comparison between MFCV tracking ASIC and $M A T L A B^{T M}$. The Mean Absolute Relative Error is presented in percentile.

TABLE IV

SUMMARY OF MFCV TRACKING ASIC.

\begin{tabular}{ll}
\hline Technology & $0.35 \mu m 2 \mathrm{P} 4 \mathrm{M}$ \\
Circuit Size & $0.954 \mathrm{~mm}^{2}$ \\
Voltage Supply & $3.3 \mathrm{~V}$ \\
Current Consumption & Total: $628 \mu \mathrm{A}$ \\
& Bit-Stream Cross-Correlator: $290 \mathrm{nA}$ \\
& Biasing Circuits: $11.5 \mu \mathrm{A}$ \\
& IA: $180 \mu \mathrm{A}$ \\
& Low-Pass Filter: $60 \mu \mathrm{A}$ \\
& Bit-Stream Converter: $68 \mu \mathrm{A}$ \\
CMRR & $85.24 \mathrm{~dB}$ \\
Gain & Adjustable (set to 111$)$ \\
Cut-off frequencies & $10.6 \mathrm{~Hz}, 2.5 \mathrm{kHz}$ internal \\
Input Referred Noise Density & $135 \mathrm{nV}$ \\
Correlation window & Adjustable by $C L K 2$ \\
Estimation Accuracy & Dependable on $C L K 1$ \\
MFCV dynamic range & Dependable on $I E D \& C L K 1$ \\
Number of Delays & 40 \\
MARD Error & $3.2 \%$ \\
\hline
\end{tabular}

\section{Discussion}

The ASIC is capable of estimating MFCV with a mean relative error of $3.2 \%$ compared to Matlab analysis. A large proportion of the error can be attributed to the nature of the bit-stream cross-correlator, as it resembles a time-to-digital converter. Hence, some error arises by quantising in time with limited resolution, as the bit stream cross-correlator has a total of 40 quantisation values. Furthermore, misalignment between ASIC output data and MATLAB estimations gives rise to unexpected error. Nevertheless, the system provides good estimation accuracy which is suitable for the purposes of muscle fatigue monitoring. Increasing the number of delays (above 40) will yield a wider dynamic range and allow higher sampling clock rate thus achieving higher estimation accuracy. This however will have a direct impact on the ASIC size.

The systems performance specifications are summarized in Table IV. The MFCV tracking ASIC realises a real time crosscorrelator with a very small footprint of $0.55 \mathrm{~mm}^{2}$ and an overall size of less than $1 \mathrm{~mm}^{2}$. Furthermore, it offers a large degree of freedom by allowing user flexibility on the correlation time window, sampling frequency and amplifier gain. With a low power consumption of $628 \mu \mathrm{A}$, the system is particularly suitable for wearable fatigue monitors. Implementation of the sEMG cross-correlator using a low power micro-controller (Nordic Semiconductor nRF51822, $4 k H z$ ADC) yielded a significantly larger dynamic power consumption of $4.8 \mathrm{~mA}$. Implementing the MFCV tracking algorithm in an ASIC enables the reduction of data transmission power costs, as well as area, without the need for EMG compression algorithms [38], [39]. Moreover, wearable devices benefit from integrated, low power solutions, allowing portability and prolonged use. The resulting small error of the proposed system supports that the system is capable of accurately estimating MFCV during static contractions.

\section{CONCLUSION}

This paper presented a first of its kind MFCV tracking ASIC and its detailed implementation and operation. Furthermore, this paper evaluates a novel algorithm for the estimation of MFCV using a single threshold converter. The ASIC is capable of tracking MFCV from surface EMG signals, using standard commercial surface electrodes. The system utilizes a sEMG Instrumentation Amplifier, a filtering stage, a bit-stream converter and a bit-stream cross-correlator. A test group of 20 people was used to demonstrate the ability of the proposed bit-stream correlator to accurately estimate MFCV in real time during static fatiguing contractions. The ASIC has a MARD error of $3.2 \%$ compared to Matlab analysis for the same dataset. The system draws $628 \mu A$ from a $3.3 \mathrm{~V}$ power supply and is implemented in a commercially available 0.35 $\mu m$ CMOS technology. The proposed ASIC is thus ideally suited for wearable muscle fatigue monitoring platforms in biomedical and clinical applications.

\section{REFERENCES}

[1] M. B. Reaz, M. Hussain, and F. Mohd-Yasin, "Techniques of emg signal analysis: detection, processing, classification and applications," Biological procedures online, vol. 8, no. 1, pp. 11-35, 2006. 
[2] D. A. Gabriel, J. R. Basford, and K.-N. An, "Assessing fatigue with electromyographic spike parameters," Engineering in Medicine and Biology Magazine, IEEE, vol. 20, no. 6, pp. 90-96, 2001.

[3] M. M. Lowery, C. L. Vaughan, P. J. Nolan, and M. J. O'Malley, "Spectral compression of the electromyographic signal due to decreasing muscle fiber conduction velocity," Rehabilitation Engineering, IEEE Transactions on, vol. 8, no. 3, pp. 353-361, 2000.

[4] G. Dimitrov, Z. Lateva, and N. Dimitrova, "Effects of changes in asymmetry, duration and propagation velocity of the intracellular potential on the power spectrum of extracellular potentials produced by an excitable fiber.," Electromyography and clinical neurophysiology, vol. 28, no. 2-3, p. 93,1988

[5] N. Dimitrova and G. Dimitrov, "Interpretation of emg changes with fatigue: facts, pitfalls, and fallacies," Journal of Electromyography and Kinesiology, vol. 13, no. 1, pp. 13-36, 2003.

[6] L. Arendt-Nielsen and K. Mills, "The relationship between mean power frequency of the emg spectrum and muscle fibre conduction velocity," Electroencephalography and clinical Neurophysiology, vol. 60, no. 2 , pp. 130-134, 1985.

[7] R. Merletti, M. Knaflitz, and C. J. De Luca, "Myoelectric manifestations of fatigue in voluntary and electrically elicited contractions," J Appl Physiol, vol. 69, no. 5, pp. 1810-20, 1990.

[8] W. H. Linssen, D. F. Stegeman, E. M. Joosten, S. L. Notermans, M. A. van't Hof, and R. A. Binkhorst, "Variability and interrelationships of surface emg parameters during local muscle fatigue," Muscle \& nerve, vol. 16, no. 8, pp. 849-856, 1993.

[9] F. B. Stulen and C. J. De Luca, "Frequency parameters of the myoelectric signal as a measure of muscle conduction velocity," Biomedical Engineering, IEEE Transactions on, no. 7, pp. 515-523, 1981.

[10] M. Zwarts, T. Van Weerden, and H. Haenen, "Relationship between average muscle fibre conduction velocity and emg power spectra during isometric contraction, recovery and applied ischemia," European journal of applied physiology and occupational physiology, vol. 56, no. 2, pp. 212-216, 1987.

[11] A. Fuglsang-Frederiksen, "The utility of interference pattern analysis," Muscle \& nerve, vol. 23, no. 1, pp. 18-36, 2000.

[12] T. Masuda, H. Miyano, and T. Sadoyama, "The measurement of muscle fiber conduction velocity using a gradient threshold zero-crossing method," Biomedical Engineering, IEEE Transactions on, no. 10, pp. 673-678, 1982.

[13] K. Masuda, T. Masuda, T. Sadoyama, M. Inaki, and S. Katsuta, "Changes in surface emg parameters during static and dynamic fatiguing contractions," Journal of Electromyography and Kinesiology, vol. 9, no. 1, pp. 39-46, 1999.

[14] D. Farina and R. Merletti, "Methods for estimating muscle fibre conduction velocity from surface electromyographic signals," Medical and biological Engineering and Computing, vol. 42, no. 4, pp. 432-445, 2004.

[15] E. Koutsos and P. Georgiou, "An analogue instantaneous median frequency tracker for emg fatigue monitoring," in Circuits and Systems (ISCAS), 2014 IEEE International Symposium on, pp. 1388-1391, IEEE, 2014.

[16] G. McVicar and P. Parker, "Spectrum dip estimator of nerve conduction velocity," Biomedical Engineering, IEEE Transactions on, vol. 35, no. 12, pp. 1069-1076, 1988.

[17] P. Parker and R. Scott, "Statistics of the myoelectric signal from monopolar and bipolar electrodes," Medical and biological engineering, vol. 11, no. 5, pp. 591-596, 1973.

[18] T. Sadoyama, T. Masuda, and H. Miyano, "Relationships between muscle fibre conduction velocity and frequency parameters of surface emg during sustained contraction," European Journal of Applied Physiology and Occupational Physiology, vol. 51, no. 2, pp. 247-256, 1983.

[19] S. Andreassen and L. Arendt-Nielsen, "Muscle fibre conduction velocity in motor units of the human anterior tibial muscle: a new size principle parameter.," The Journal of Physiology, vol. 391, no. 1, pp. 561-571, 1987.

[20] T. Sadoyama, T. Masuda, H. Miyata, and S. Katsuta, "Fibre conduction velocity and fibre composition in human vastus lateralis," European journal of applied physiology and occupational physiology, vol. 57, no. 6 , pp. 767-771, 1988.

[21] L. Arendt-Nielsen and K. R. Mills, "Muscle fibre conduction velocity, mean power frequency, mean emg voltage and force during submaximal fatiguing contractions of human quadriceps," European journal of applied physiology and occupational physiology, vol. 58, no. 1-2, pp. 20 25, 1988.

[22] X. Ye, T. Beck, and N. Wages, "Relationship between innervation zone width and mean muscle fiber conduction velocity during a sustained iso- metric contraction," Journal of musculoskeletal \& neuronal interactions, vol. 15, no. 1, pp. 95-102, 2015.

[23] D. Farina, M. Pozzo, E. Merlo, A. Bottin, and R. Merletti, "Assessment of average muscle fiber conduction velocity from surface emg signals during fatiguing dynamic contractions," Biomedical Engineering, IEEE Transactions on, vol. 51, no. 8, pp. 1383-1393, 2004.

[24] E. M. Spinelli, R. Pallas-Areny, and M. A. Mayosky, "Ac-coupled frontend for biopotential measurements," Biomedical Engineering, IEEE Transactions on, vol. 50, no. 3, pp. 391-395, 2003.

[25] R. F. Yazicioglu, S. Kim, T. Torfs, H. Kim, and C. Van Hoof, "A 30 w analog signal processor asic for portable biopotential signal monitoring," Solid-State Circuits, IEEE Journal of, vol. 46, no. 1, pp. 209-223, 2011.

[26] N. Verma, A. Shoeb, J. Bohorquez, J. Dawson, J. Guttag, and A. P. Chandrakasan, "A micro-power eeg acquisition soc with integrated feature extraction processor for a chronic seizure detection system," Solid-State Circuits, IEEE Journal of, vol. 45, no. 4, pp. 804-816, 2010.

[27] R. R. Harrison and C. Charles, "A low-power low-noise cmos amplifier for neural recording applications," Solid-State Circuits, IEEE Journal of, vol. 38, no. 6, pp. 958-965, 2003

[28] R. Yazicioglu, P. Merken, R. Puers, and C. Van Hoof, "A 60 uw 60 $\mathrm{nv} / \mathrm{hz}$ readout front-end for portable biopotential acquisition systems," Solid-State Circuits, IEEE Journal of, vol. 42, no. 5, pp. 1100-1110, 2007.

[29] R. F. Yazicioglu, P. Merken, R. Puers, and C. Van Hoof, "A 200 w eight-channel eeg acquisition asic for ambulatory eeg systems," SolidState Circuits, IEEE Journal of, vol. 43, no. 12, pp. 3025-3038, 2008.

[30] T. Denison, K. Consoer, W. Santa, A.-T. Avestruz, J. Cooley, and A. Kelly, "A 2 uw $100 \mathrm{nv} / \mathrm{rthz}$ chopper-stabilized instrumentation amplifier for chronic measurement of neural field potentials," Solid-State Circuits, IEEE Journal of, vol. 42, no. 12, pp. 2934-2945, 2007.

[31] C. J. De Luca, L. Donald Gilmore, M. Kuznetsov, and S. H. Roy, "Filtering the surface emg signal: Movement artifact and baseline noise contamination," Journal of biomechanics, vol. 43, no. 8, pp. 1573-1579, 2010.

[32] G. Kamen and D. Gabriel, Essentials of electromyography. Human Kinetics, 2010.

[33] T. S. Lande, T. G. Constandinou, A. Burdett, and C. Toumazou, "Running cross-correlation using bitstream processing," Electronics letters, vol. 43, no. 22, pp. 1181-1183, 2007.

[34] T.-D. Chiueh, P.-Y. Tsai, and I.-W. Lai, Baseband Receiver Design for Wireless MIMO-OFDM Communications. John Wiley \& Sons, 2012.

[35] S. Henzler, "Time-to-digital converter basics," in Time-to-Digital Converters, pp. 5-18, Springer, 2010.

[36] D. Farina, E. Fortunato, and R. Merletti, "Noninvasive estimation of motor unit conduction velocity distribution using linear electrode arrays," Biomedical Engineering, IEEE Transactions on, vol. 47, no. 3, pp. 380$388,2000$.

[37] P. A. Lynn, "Direct on-line estimation of muscle fiber conduction velocity by surface electromyography," Biomedical Engineering, IEEE Transactions on, no. 10, pp. 564-571, 1979.

[38] A. M. Dixon, E. G. Allstot, D. Gangopadhyay, and D. J. Allstot, "Compressed sensing system considerations for ecg and emg wireless biosensors," Biomedical Circuits and Systems, IEEE Transactions on, vol. 6, no. 2, pp. 156-166, 2012.

[39] J. Zhang, Y. Suo, S. Mitra, S. P. Chin, S. Hsiao, R. F. Yazicioglu, T. D. Tran, and R. Etienne-Cummings, "An efficient and compact compressed sensing microsystem for implantable neural recordings," Biomedical Circuits and Systems, IEEE Transactions on, vol. 8, no. 4, pp. 485496, 2014.

[40] M. Naeije, "Estimation of the action potential conduction velocity in human skeletal muscle using the surface emg cross-correlation technique," Electromyogr Clin Neurophysiol, vol. 23, pp. 73-80, 1983. 


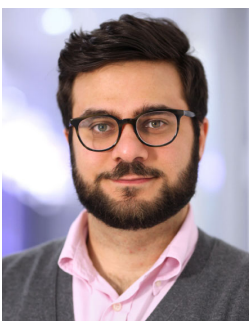

Ermis Koutsos (S'12) received his BSc degree in Electrical and Electronic Engineering from University of Surrey - U.K., in 2011 and his MSc in analogue and digital integrated circuit design from the Dept. of Electrical and Electronic Engineering of Imperial College London - U.K., in 2012. Since 2012 he is a $\mathrm{PhD}$ candidate in the Centre of Bio-Inspired Technology, Institute of Biomedical Engineering, Imperial College London, under the supervision of Dr. Pantelis Georgiou. His research interest focuses on the design of low power mixed signal electronics for biomedical applications. Mr. Koutsos is a scholar of EPSRC.

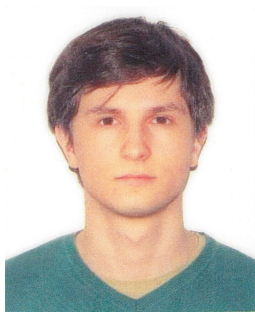

Vlad Cretu received the B.S. degree in electrical engineering from the University Pierre et Marie Curie, Paris, France in 2013 and the M.Sc degree in analogue and digital integrated circuit design from Imperial College, London, UK, in 2014. In 2014, he joined Sensata Technologies Swindon Silicon Systems as a design engineer. His main field of interest is high performance CMOS with biomedical applications.

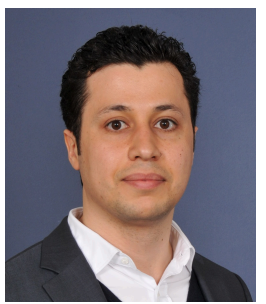

Pantelis Georgiou (AM'05-M'08-SM'13) received the M.Eng. degree in Electrical and Electronic Engineering in 2004 and the Ph.D. degree in 2008 both from Imperial College London. He is currently a lecturer within the Department of Electrical \& Electronic Engineering and is also the head of the Bio-inspired Metabolic Technology Laboratory in the Centre for Bio-Inspired Technology and part of the Medical Engineering Solutions in Osteoarthritis Centre of Excellence.

His research includes bio-inspired circuits and systems, CMOS based lab-on-chip technologies and application of microelectronic technology to create novel medical devices. He conducted pioneering work on the silicon beta cell and is now leading the project forward to the development of the first bio-inspired artificial pancreas for Type I diabetes. In 2013 he was awarded the IET Mike Sergeant medal of outstanding contribution to engineering. Dr Georgiou is a member of the IEEE and IET and serves on the BioCAS and Sensory Systems technical committees of the IEEE CAS Society. He also serves on the IET Prizes and Awards committee. 